\title{
EFFECT OF SOME POSTHARVEST TREATMENTS ON FRUIT QUALITY AND STORABILITY OF WINTER GUAVA (PSIDIUM GUAJAVA L.) ETMANY STRAIN
}

\author{
M. A. A MOHAMED* and ZEINAB. A. ZAKI* \\ *Fruit Handling Department, Hortculture Reserch Institute, ARC, Giza, Egypt.
}

(Manuscript received 28 March 2018)

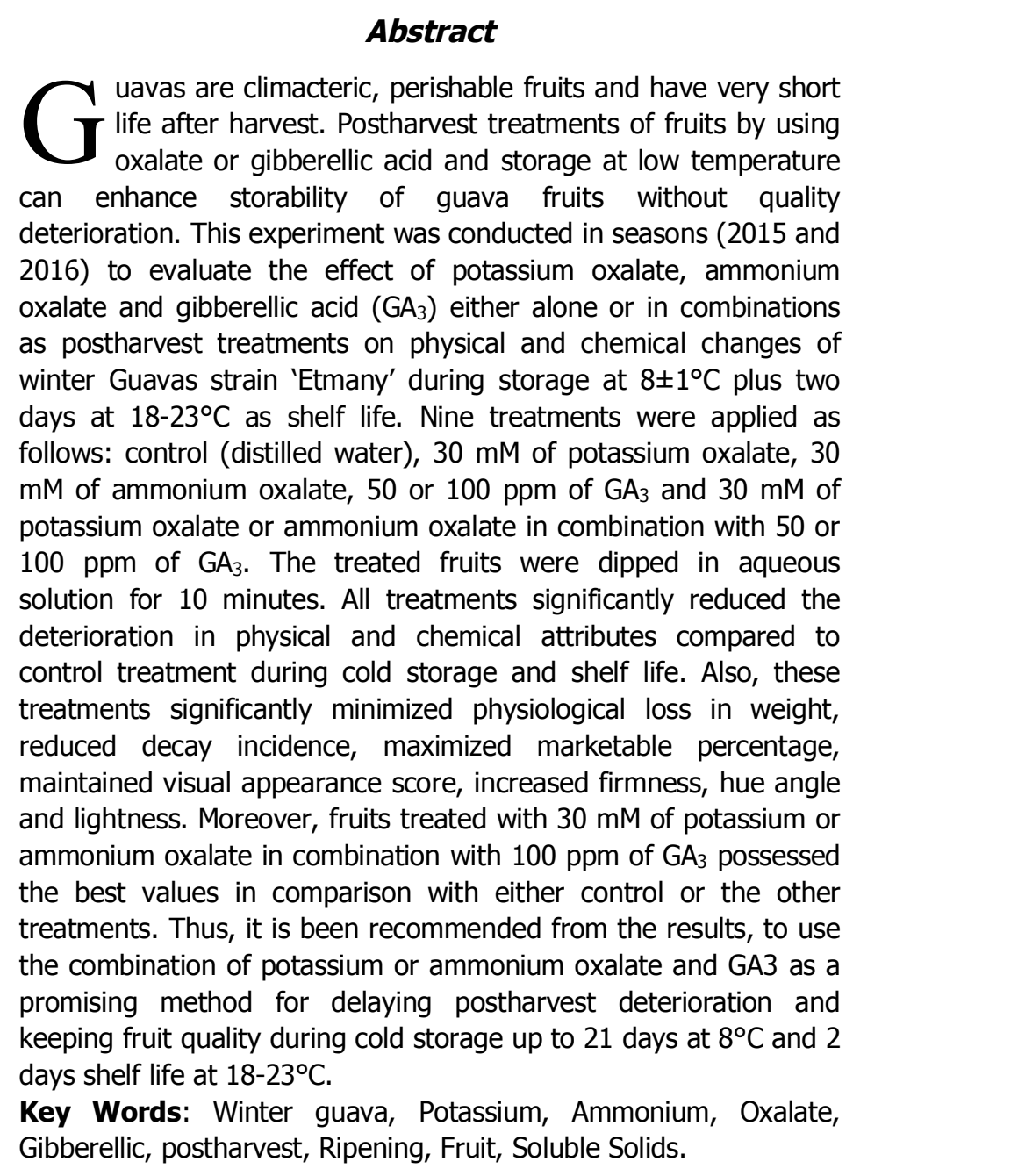

\section{INTRODUCTION}

Guava (Psidium guajava L.) is one of the most well known edible fruits grown widely throughout tropical and subtropical regions of the world. Guava fruits expressed as the main source of vitamins A, B, C and minerals (Kumar, 2012). It is very popular to the Egyptian consumer due to its suitable price and high nutritional value. In Egypt, the total guava area reached 36529 feddans, 33706 feddans of them are fruiting trees produced 343702 tons according to the Ministry of agriculture and land reclamation statistics, Anonymous, 2016. One of the major drawbacks of fresh 
guava is that it bruise easily and are highly perishable, which leads to mechanical injuries and high incidence of fungi rotting and shriveling. Thereby, fruits cannot be stored for a long time (Kader, 2002). Guava is a climacteric fruits and has poor storage life after harvest. Due to fast ripening caused by the rise in ethylene production parallels a peak in respiration rate, which greatly affects a loss in Guava fruits commercial value. Hence, the reduction in temperature after harvest is necessary to delay the climacteric peak and consequently ripening process (Kader, 2002). Storage life enhancement of guavas could reduce losses percent, maintain fruit quality and regulate the supply of guavas for long period. Therefore, it is necessary to find a feasible solution to slow down the action of ethylene gas to control the ripening of guava fruit after harvest. Fortunately, various methods used to improve and maintain quality by slowing down the metabolic activities of fruits.

Gibberellins are a group of growth substances, known to delay ripening and acts as anti-senescence agent during storage (Hooley, 1994). Gibberellic acid (GA3) is one of an ethylene antagonist, approved by the United States Environmental Protection Agency. Moreover, $\mathrm{GA}_{3}$ could retard ripening and senescence processes of fruits through post-harvest treatments by delaying incidence of respiration rate peak, and subsequently ethylene production (Reena, 2016), delaying the chlorophyll degradation, softening and retaining the internal fruit quality by slowing down the metabolic activities of guava fruits (Reena, 2016).

In addition, potassium and ammonium oxalate postharvest treatments could be promising materials to reduce decay incidence and delay ripening process of mangoes at room temperature (Zheng et al., 2012).

Winter crop of guava fruits are free from fruit fly infestation and fetch higher price as compared to main season crops (Boora et al., 2016). The reduction in losses and extension of postharvest life of guava fruits will help to increase the market price. So, the aim of this study was to examine the effect of gibberellic acid, potassium oxalate and ammonium oxalate applied either alone or in combinations on postharvest quality, decay incidence and storability of winter guava fruits strain 'Etmany' under cold storage conditions at $8^{\circ} \mathrm{C} \pm 1^{\circ} \mathrm{C}$ and $90 \pm 5 \%$ relative humidity (RH).

\section{MATERIALS AND METHODS}

The present experiment conducted during two successive seasons (2015 and 2016). Winter guava fruit cv. 'Etmany' were picked at maturity stage (yellowish green) on the second week of February according to (Mercado-Silva et al., 1998). Guava trees grown in a commercial orchard in El-Qalubia Governorate, Egypt (latitude, $30^{\circ} 17^{\prime \prime} \mathrm{N}$ and longitude, $31^{\circ} 20^{\prime \prime} \mathrm{E}$ ). The trees were about 10 years of age and planted 
at a spacing of $5 \times 5$ meters apart in loamy clay soil under immersion irrigation system and subjected to all ideal agriculture practices. Fruits were almost similar, apparently uniform in size and free of visible symptoms of infection. In both experimental seasons, fruits were harvested, picked in plastic boxes (15 kg capacity), and immediately brought to postharvest laboratory at Horticulture Research Institute, Agriculture Research Center, Giza governorate, nearly at 50 kilometers from the orchard. At the laboratory, all defective fruits including wounded and other disorders were excluded. The sound fruits at the same maturity stage were washed with tap water solution for 2 min to clean the surface of fruits and then air dried at room temperature until visible moisture on fruit surfaces disappeared completely. Clean sound fruits were randomly selected and divided into nine groups. Each treatment consisted of 144 fruits with three replicates for each examination period and each replicate contained 12 individual fruit.

\section{The treatments:-}

Guava fruits were immersed into one of an aqueous solution as the following:-

\begin{tabular}{|l|l|l|l|}
\hline T nu & Treatments & T nu & Treatments \\
\hline 1 & $30 \mathrm{mM}$ ammonium oxalate $(\mathrm{AO})$ & 2 & $30 \mathrm{mM}$ potassium oxalate $(\mathrm{PO})$ \\
\hline 3 & $50 \mathrm{ppm}$ gibberellic acid $\left(\mathrm{GA}_{3}\right)$ & 4 & $100 \mathrm{ppm} \mathrm{GA}$ \\
\hline 5 & $30 \mathrm{mM}$ AO plus $50 \mathrm{ppm} \mathrm{GA}$ & 6 & $30 \mathrm{mM} \mathrm{AO}$ plus $100 \mathrm{ppm} \mathrm{GA}$ \\
\hline 7 & $30 \mathrm{mM}$ PO plus $50 \mathrm{ppm} \mathrm{GA} A_{3}$ & 8 & $30 \mathrm{mM}$ PO plus $100 \mathrm{ppm} \mathrm{GA}$ \\
\hline 9 & Distilled water (control) & & \\
\hline
\end{tabular}

Guava fruits were immersed in five liter of an aqueous solution containing Tween-80 $0.05 \%(v / v)$ to improve wettability and adherence to guavas surface for ten minutes. After dipping treatments, fruits were air dried for $30 \mathrm{~min}$ at room temperature by electric fan. All fruits were placed into unsealed plastic bags with a thickness of $0.04 \mathrm{~mm}$ and each bag contained 6 individual fruit with 24 bags per treatment and then packaged in cardboard boxes with dimensions of $45 \times 35 \times 10 \mathrm{~cm}$. Experimental boxes were stored at $8 \pm 1^{\circ} \mathrm{C}$ and $90 \pm 5 \% \mathrm{RH}$ for 21 days. Each treatment was examined at harvest time and each seven days intervals to examine the effect of postharvest treatments on physical and chemical properties of guava fruits.

\subsection{The Physical Characteristics Measurements}

2.1.1. Weight loss percentage was calculated by the following equation: [(A $B) / A \times 100]$, where $A$ is the initial fruit weight and $B$ is the fruit weight after each period of cold storage.

2.1.2. Decay percentage was recorded by the weight of decayed fruits due to fungus or any microorganisms infection and calculated as a percentage of the initial 
weight of fruits using the following formula: $[(C / A) \times 100]$, where $A$ is the initial fruit weight and $\mathrm{C}$ is the decayed fruit weight after each period of cold storage.

2.1.3. Marketable fruits percentage was calculated by the following equation: $[(D / A) \times 100]$, where $A$ is the fruit weight before storage and $D$ is the sound fruit weight after special storage period.

2.1.4.Visual appearance was measured by a rating system and fruit was scored as very good $=9$, good $=7$, acceptable $=5$, unacceptable $=3$ and poor $=1$

2.1.4. Skin colour was measured by using a Minolta CR-400 Chroma Meter (Minolta Co. Itd. Osaka, Japan). The skin colour measurement was expressed in chromaticity values of lightness $(L)$ and hue angel $\left(h^{\circ}\right)$. Three readings were taken at different locations of each fruit during each data observation according to (McGuire, 1992).

2.1.5. Fruit firmness was measured in three guava fruits per replication at two equatorial sites by using penetrometer (Effegi hand-held, facchini, Alfonsine, Italy) equipped with an $8 \mathrm{~mm}$ cylindrical stainless steel plunger tip (Watkins and Harman, 1981). The firmness value was expressed in terms of $\mathrm{lb} / \mathrm{inch}^{2}$ and data was calculated as Newton/ $/ \mathrm{cm}^{2}$ by the following formula ( 1 Newton $\left./ \mathrm{cm}^{2}=1.45 \mathrm{lb} / \mathrm{inch}^{2}\right)$.

\subsection{The Chemical Characteristics Measurements}

The juice extracted by crushing the pulp of guava fruit then filtered through a muslin cloth and used for measuring the following:

2.2.1. Vitamin C value which was estimated according to (AOAC, 2000) and presented as $\mathrm{mg} / 100 \mathrm{ml}$ of the juice.

2.2.2. Total soluble solids (TSS) and Titratable acidity (TA) content were measured using a hand refractometer for TSS and as grams of citric acid per $100 \mathrm{ml}$ of juice for acidity according to (AOAC, 2000).

2.2.3. TSS/TA ratio was calculated by dividing the TSS values by total acidity.

\subsection{Statistical analysis}

This experiment was arranged in a completely randomized design. Recorded data were analyzed statistically by analysis of variance (ANOVA) using the MSTAT-C statistical package (M-STAT, 1993). Means comparison were done by Duncan's multiple range tests (DMRT) at probability $\leq 0.05$.

\section{RESULTS AND DISCUSSION}

\subsection{Influence of postharvest treatments on fruits quality and storability of winter guava fruits strain 'Etmany' during storage:-}




\subsubsection{Physical characteristics:}

\section{Weight loss, decay, marketable percentage and visual appearance}

In this experiment, data in Tables (1, 2, 3 and 4) revealed that, all studied postharvest treatments significantly decreased fruit weight loss and decay percentage of Guavas strain 'Etmany' than control (untreated) fruits. On the other hand, treated fruits showed a significant increase in marketable percentage and visual appearance as compared to control treatment in both seasons. In addition, fruits that dipped in potassium or ammonium oxalate at $30 \mathrm{mM}$ combined with gibberellic acid at 50 or 100 ppm concentration for 10 min were more effective in reducing weight loss and decay percentage compared with other treatments.

Data also declared that, Fruits treated with $30 \mathrm{mM}$ of potassium oxalate in combination with gibberellic acid at $100 \mathrm{ppm}$ followed by $30 \mathrm{mM}$ of ammonium oxalate in combination with gibberellic acid at $100 \mathrm{ppm}$ possessed the highest effectiveness in reducing fruits deterioration i.e. fruit weight, marketable percentage and visual appearance score, and the lowest decay incidence during storage in the two seasons compared with control treatments.

Data in table 1 indicated that fruits treated with $30 \mathrm{mM}$ of potassium oxalate plus gibberellic acid at $100 \mathrm{ppm}$ recorded the lowest significant weight loss (2.92 \& $4.15 \%)$ and decay incidence ( $3.10 \& 3.82 \%)$ and recorded the highest marketable fruits (94.15 \& $92.30 \%)$ and visual appearance score $(8.75 \& 8.67)$ in the first season and the second season, respectively. On the other hand, control (untreated) fruits exhibited the highest weight loss values (10.28 \& $11.62 \%)$ and decay incidence $(15.50 \& 18.30 \%)$ and exhibited the lowest marketable fruits $(76.44 \& 72.87 \%)$ and visual appearance score $(5.50 \& 5.17)$ in both seasons, respectively.

With respect to storage period effect, data in Tables (1, 2, 3 and 4) demonstrated that weight loss and decay percentage were increased gradually and significantly with prolonging of storage period in both seasons. On contrast, guava fruits showed gradual and significant reduction in marketable percentage and visual appearance with the advancement of storage period during the two seasons.

The interaction effect between pre-storage treatments and storage periods showed significant differences at $(p \leq 0.05)$ for these parameters.

The increasing in weight loss and decay incidence may be due to the increase in respiration, water evaporation, ripening and senescence process during storage and shelf life (Zheng et al., 2012 and Reena, 2016).

Potassium or ammonium oxalate combined with gibberellic acid reduced the weight loss and decay percentage, this reduction might be due to its anti-senescence, 
and anti-transpiration properties that cause the decrease in the tissue permeability, reduce cellular metabolic activities. Hence reducing the physiological loss in weight with enhancing resistant ability in the fruit leading to the delay in fruit ripening as mentioned by (Zheng et al., 2007; Zheng et al., 2012 and Reena, 2016).

Previous work has showed that pre-storage potassium and ammonium oxalate treatments increased disease resistance of mangoes (Zheng et al.,2012) by increasing antioxidant activity, thus extended the storage time and decreased the decay incidence during storage.

These results are in harmony with the findings of (Reena, 2016) on guavas and (Zheng et al., 2012) on mangoes who indicated that, oxalate and gibberellic acid postharvest treatments reduced weight loss percentage and decay incidence with an increase in marketable fruit percentage during storage and shelf life.

Table 1. Influence of some postharvest treatments on weight loss percentage of guava fruits strain 'Etmany' under cold storage conditions during 2015 and 2016 seasons.

\begin{tabular}{|c|c|c|c|c|c|c|}
\hline \multirow{3}{*}{ Postharvest treatments } & \multicolumn{4}{|c|}{ Storage period (day) } & & \\
\hline & $0+2$ & $7+2$ & $14+2$ & $21+2$ & \multirow{2}{*}{\multicolumn{2}{|c|}{ Means }} \\
\hline & \multicolumn{4}{|c|}{2015 Season } & & \\
\hline Distilled water (control) & $2.67 \mathrm{klm}$ & $7.82 \mathrm{c}-\mathrm{f}$ & $12.76 \mathrm{~b}$ & $17.89 \mathrm{a}$ & 10.28 & $A$ \\
\hline $30 \mathrm{mM}$ ammonium oxalate (AO) & $1.00 \mathrm{~m}$ & $3.14 \mathrm{jkl}$ & 7.59 e-h & $10.86 \mathrm{bcd}$ & 5.65 & B \\
\hline $30 \mathrm{mM}$ potassium oxalate $(\mathrm{PO})$ & $0.73 \mathrm{~m}$ & $3.08 \mathrm{jkl}$ & 7.17 e-i & 9.03 cde & 5.00 & $\mathrm{BC}$ \\
\hline 50 ppm gibberellic acid $\left(\mathrm{GA}_{3}\right)$ & $1.03 \mathrm{~m}$ & $3.27 \mathrm{jkl}$ & 9.69 efg & $11.39 \mathrm{bc}$ & 6.35 & B \\
\hline $100 \mathrm{ppm} \mathrm{GA}$ & $0.87 \mathrm{~m}$ & $3.19 \mathrm{jkl}$ & $7.00 \mathrm{f}-\mathrm{j}$ & 9.04 cde & 5.02 & $\mathrm{BC}$ \\
\hline $30 \mathrm{mM}$ AO plus 50 ppm $\mathrm{GA}_{3}$ & $0.53 \mathrm{~m}$ & $2.48 \mathrm{klm}$ & $5.05 \mathrm{~g}-\mathrm{j}$ & 8.48 def & 4.14 & $\mathrm{CD}$ \\
\hline $30 \mathrm{mM} \mathrm{AO}$ plus 100 ppm GA 3 & $0.33 \mathrm{~m}$ & $2.40 \mathrm{klm}$ & $3.28 \mathrm{i}-\mathrm{I}$ & 7.83 efg & 3.46 & $\mathrm{D}$ \\
\hline $30 \mathrm{mM}$ PO plus 50 ppm GA & $0.47 \mathrm{~m}$ & $2.43 \mathrm{klm}$ & $4.04 \mathrm{~h}-\mathrm{k}$ & 8.22 def & 3.79 & $C D$ \\
\hline $30 \mathrm{mM}$ PO plus $100 \mathrm{ppm} \mathrm{GA_{3 }}$ & $0.20 \mathrm{~m}$ & $1.68 \mathrm{Im}$ & $3.21 \mathrm{i}-\mathrm{I}$ & 6.58 e-h & 2.92 & $\mathrm{D}$ \\
\hline \multirow[t]{2}{*}{$\begin{array}{r}\text { Means } \\
\end{array}$} & $0.87 \mathrm{D}$ & $3.28 \mathrm{C}$ & $6.64 \mathrm{~B}$ & $9.92 \mathrm{~A}$ & & \\
\hline & \multicolumn{4}{|c|}{2016 Season } & & \\
\hline Distilled water (control) & $3.25 \mathrm{ijk}$ & $10.36 \mathrm{efg}$ & $14.54 b$ & $18.33 \mathrm{a}$ & 11.62 & $A$ \\
\hline $30 \mathrm{mM}$ ammonium oxalate $(\mathrm{AO})$ & $1.59 \mathrm{jk}$ & $4.62 \mathrm{ijk}$ & 8.09 efg & $12.55 \mathrm{bcd}$ & 6.71 & $\mathrm{BC}$ \\
\hline 30 mM potassium oxalate (PO) & $1.33 \mathrm{k}$ & 4.63 ijk & 7.67 efg & $10.56 \mathrm{c}-\mathrm{f}$ & 6.05 & $\mathrm{BCD}$ \\
\hline 50 ppm gibberellic acid $\left(\mathrm{GA}_{3}\right)$ & $1.63 \mathrm{jk}$ & $4.79 \mathrm{ijk}$ & 9.17 cde & $13.07 \mathrm{bc}$ & 7.16 & B \\
\hline $100 \mathrm{ppm} \mathrm{GA}$ & $1.46 \mathrm{k}$ & $4.76 \mathrm{ijk}$ & 7.50 efg & $10.59 \mathrm{c}-\mathrm{f}$ & 6.08 & $\mathrm{BCD}$ \\
\hline $30 \mathrm{mM} \mathrm{AO}$ plus 50 ppm GA 3 & $1.13 \mathrm{k}$ & $3.36 \mathrm{ijk}$ & $6.98 \mathrm{ghi}$ & 10.11 def & 5.39 & $\mathrm{CDE}$ \\
\hline $30 \mathrm{mM} \mathrm{AO}$ plus $100 \mathrm{ppm} \mathrm{GA_{3 }}$ & $0.93 \mathrm{k}$ & $3.27 \mathrm{ijk}$ & $5.22 \mathrm{ijk}$ & 9.46 efg & 4.72 & $\mathrm{DE}$ \\
\hline $30 \mathrm{mM}$ PO plus 50 ppm $\mathrm{GA}_{3}$ & $1.06 \mathrm{k}$ & $3.35 \mathrm{ijk}$ & 6.00 hij & 9.97 def & 5.10 & $\mathrm{DE}$ \\
\hline $30 \mathrm{mM}$ PO plus 100 ppm GA 3 & $0.80 \mathrm{k}$ & $2.57 \mathrm{jk}$ & $5.07 \mathrm{ijk}$ & $8.14 \mathrm{fgh}$ & 4.15 & $\mathrm{E}$ \\
\hline Means & $1.46 \mathrm{D}$ & $4.63 \mathrm{C}$ & $7.81 \mathrm{~B}$ & $11.42 \mathrm{~A}$ & & \\
\hline
\end{tabular}

Means followed by the same letters season are not significantly different at level $P \leq 0.05$ according to DMRT. 
Table 2. Influence of some postharvest treatments on decay percentage of guava fruits strain 'Etmany' under cold storage conditions during 2015 and 2016 seasons.

\begin{tabular}{|c|c|c|c|c|c|c|c|}
\hline \multirow{3}{*}{ Postharvest treatments } & \multicolumn{5}{|c|}{ Storage period (day) } & & \\
\hline & $0+2$ & & +2 & $14+2$ & $21+2$ & \multicolumn{2}{|c|}{ Means } \\
\hline & \multicolumn{5}{|c|}{2015 Season } & & \\
\hline Distilled water (control) & $0.00 \mathrm{j}$ & 12.85 & $c-h$ & $18.40 \mathrm{~b}$ & 30.77 a & 15.50 & $A$ \\
\hline $30 \mathrm{mM}$ ammonium oxalate (AO) & $0.00 \mathrm{j}$ & 7.46 & $e-i$ & $12.20 \mathrm{c}-\mathrm{h}$ & $17.43 \mathrm{bc}$ & 9.27 & B \\
\hline 30 mM potassium oxalate (PO) & $0.00 \mathrm{j}$ & 4.84 & hij & $11.96 \mathrm{c}-\mathrm{i}$ & $15.83 \mathrm{bcd}$ & 8.16 & $\mathrm{BC}$ \\
\hline 50 ppm gibberellic acid $\left(\mathrm{GA}_{3}\right)$ & $0.00 \mathrm{j}$ & 6.67 & $f-j$ & $14.35 \mathrm{c}-\mathrm{g}$ & 18.20 bc & 9.80 & $B$ \\
\hline $100 \mathrm{ppm} \mathrm{GA}$ & $0.00 \mathrm{j}$ & 5.82 & $g-j$ & $12.13 \mathrm{c}-\mathrm{h}$ & $16.22 \mathrm{bcd}$ & 8.54 & B \\
\hline $30 \mathrm{mM}$ AO plus 50 ppm $\mathrm{GA}_{3}$ & $0.00 \mathrm{j}$ & 0.00 & j & $6.72 \mathrm{f}-\mathrm{j}$ & 12.47 b-e & 4.80 & $C D$ \\
\hline $30 \mathrm{mM} \mathrm{AO}$ plus $100 \mathrm{ppm} \mathrm{GA}_{3}$ & $0.00 \mathrm{j}$ & 0.00 & j & $5.67 \mathrm{hij}$ & $10.54 \mathrm{c}-\mathrm{h}$ & 4.05 & D \\
\hline $30 \mathrm{mM}$ PO plus 50 ppm GA 3 & $0.00 \mathrm{j}$ & 0.00 & j & $6.45 \mathrm{~g}-\mathrm{j}$ & $12.85 b-f$ & 4.83 & $C D$ \\
\hline $30 \mathrm{mM}$ PO plus 100 ppm GA & $0.00 \mathrm{j}$ & 0.00 & j & $4.38 \mathrm{ij}$ & $8.02 \mathrm{~d}-\mathrm{i}$ & 3.10 & $\mathrm{D}$ \\
\hline \multirow[t]{2}{*}{ Means } & $0.00 \mathrm{D}$ & 4.18 & $\mathrm{C}$ & $10.25 \mathrm{~B}$ & $15.81 \mathrm{~A}$ & & \\
\hline & \multicolumn{5}{|c|}{2016 Season } & & \\
\hline Distilled water (control) & $0.00 \mathrm{~g}$ & 14.23 & b-e & $23.25 \mathrm{~b}$ & 35.73 a & 18.30 & $A$ \\
\hline $30 \mathrm{mM}$ ammonium oxalate (AO) & $0.00 \mathrm{~g}$ & 9.12 & $d-g$ & $13.43 b-f$ & $19.69 \mathrm{~b}$ & 10.56 & B \\
\hline 30 mM potassium oxalate (PO) & $0.00 \mathrm{~g}$ & 6.29 & efg & $13.16 \mathrm{~b}-\mathrm{f}$ & 17.42 bc & 9.22 & $\mathrm{BC}$ \\
\hline 50 ppm gibberellic acid (GA3) & $0.00 \mathrm{~g}$ & 7.80 & $d-g$ & $14.92 \mathrm{bcd}$ & $20.04 b$ & 10.69 & B \\
\hline 100 ppm GA3 & $0.00 \mathrm{~g}$ & 7.40 & efg & $13.35 b-f$ & $18.32 \mathrm{~b}$ & 9.77 & B \\
\hline 30 mM AO plus 50 ppm GA3 & $0.00 \mathrm{~g}$ & 0.00 & $\mathrm{~g}$ & $7.51 \mathrm{~d}-\mathrm{g}$ & $16.13 b-f$ & 5.91 & $C D$ \\
\hline $30 \mathrm{mM}$ AO plus 100 ppm GA3 & $0.00 \mathrm{~g}$ & 0.00 & $g$ & 6.34 efg & $14.01 b-f$ & 5.09 & $D$ \\
\hline 30 mM PO plus 50 ppm GA3 & $0.00 \mathrm{~g}$ & 0.00 & $g$ & $7.21 \mathrm{~d}-\mathrm{g}$ & $15.66 \mathrm{~b}-\mathrm{e}$ & 5.72 & $C D$ \\
\hline 30 mM PO plus 100 ppm GA3 & $0.00 \mathrm{~g}$ & 0.00 & $g$ & $5.07 \mathrm{fg}$ & $10.19 \mathrm{c}-\mathrm{f}$ & 3.82 & $D$ \\
\hline Means & $0.00 \mathrm{D}$ & 4.98 & $\mathrm{C}$ & $11.58 \mathrm{~B}$ & $18.58 \mathrm{~A}$ & & \\
\hline
\end{tabular}

Means followed by the same letters season are not significantly different at level $\mathrm{P} \leq 0.05$ according to DMRT

Table 3. Influence of some postharvest treatments on marketable percentage of guava fruits strain 'Etmany' under cold storage conditions during 2015 and 2016 seasons.

\begin{tabular}{|c|c|c|c|c|c|c|c|c|c|c|}
\hline \multirow{3}{*}{ Postharvest treatments } & \multicolumn{8}{|c|}{ Storage period (day) } & & \\
\hline & \multicolumn{2}{|c|}{$0+2$} & \multicolumn{2}{|r|}{$7+2$} & \multicolumn{2}{|c|}{$14+2$} & \multicolumn{2}{|c|}{$21+2$} & \multicolumn{2}{|c|}{ Means } \\
\hline & \multicolumn{8}{|c|}{2015 Season } & & \\
\hline Distilled water (control) & 97.33 & $a b c$ & 80.49 & hij & 71.07 & 1 & 56.87 & $\mathrm{~m}$ & 76.44 & $D$ \\
\hline $30 \mathrm{mM}$ ammonium oxalate (AO) & 99.00 & $\mathrm{a}$ & 89.65 & $d-g$ & 81.12 & ghi & 74.09 & jkl & 85.96 & $B C$ \\
\hline 30 mM potassium oxalate (PO) & 99.27 & $a$ & 92.20 & $\mathrm{~cd}$ & 82.02 & $e-i$ & 78.27 & hij & 87.94 & B \\
\hline 50 ppm gibberellic acid $\left(\mathrm{GA}_{3}\right)$ & 98.97 & $a$ & 90.32 & de & 77.54 & hij & 71.23 & $\mathrm{kl}$ & 84.51 & C \\
\hline 100 ppm GA 3 & 99.13 & $a$ & 91.18 & $d$ & 81.60 & $f-i$ & 76.41 & ijk & 87.08 & $\mathrm{BC}$ \\
\hline $30 \mathrm{mM} \mathrm{AO}$ plus 50 ppm GA & 99.47 & a & 97.52 & $a b c$ & 88.40 & $d-g$ & 80.10 & hij & 91.37 & A \\
\hline $30 \mathrm{mM} \mathrm{AO}$ plus $100 \mathrm{ppm} \mathrm{GA}_{3}$ & 99.67 & $a$ & 97.60 & $a b c$ & 91.24 & $d$ & 82.47 & hij & 92.74 & A \\
\hline $30 \mathrm{mM}$ PO plus 50 ppm GA & 99.53 & $\mathrm{a}$ & 97.57 & $a b c$ & 89.76 & def & 79.98 & hij & 91.71 & $A$ \\
\hline $30 \mathrm{mM}$ PO plus 100 ppm GA & 99.80 & $\mathrm{a}$ & 98.32 & $a b$ & 92.57 & bcd & 85.90 & $d-h$ & 94.15 & A \\
\hline \multirow[t]{2}{*}{ Means } & 99.13 & $A$ & 92.76 & B & 83.92 & C & 76.15 & D & & \\
\hline & \multicolumn{8}{|c|}{2016 Season } & & \\
\hline Distilled water (control) & 96.75 & $a b c$ & 76.65 & fgh & 65.55 & $\mathrm{i}$ & 52.53 & j & 72.87 & $\mathrm{D}$ \\
\hline $30 \mathrm{mM}$ ammonium oxalate (AO) & 98.41 & $a b$ & 86.70 & d & 79.55 & fgh & 70.77 & hi & 83.86 & $\mathrm{C}$ \\
\hline 30 mM potassium oxalate (PO) & 98.67 & $a b$ & 89.35 & bcd & 80.49 & efg & 75.75 & gh & 86.06 & $B C$ \\
\hline 50 ppm gibberellic acid $\left(\mathrm{GA}_{3}\right)$ & 98.37 & $a b$ & 87.82 & $d$ & 77.63 & ghi & 68.13 & i & 82.99 & C \\
\hline $100 \mathrm{ppm} \mathrm{GA}$ & 98.54 & $a b$ & 88.19 & $\mathrm{~cd}$ & 80.01 & $\mathrm{fg}$ & 73.26 & ghi & 85.00 & C \\
\hline $30 \mathrm{mM} \mathrm{AO}$ plus 50 ppm GA & 98.87 & $a b$ & 96.64 & $a b c$ & 85.86 & de & 75.39 & fgh & 89.19 & $A B$ \\
\hline $30 \mathrm{mM} \mathrm{AO}$ plus 100 ppm GA & 99.07 & a & 96.73 & $a b c$ & 88.77 & cd & 77.87 & efg & 90.61 & A \\
\hline 30 mM PO plus 50 ppm GA & 98.94 & $a b$ & 96.65 & $a b c$ & 87.21 & $d$ & 75.90 & fgh & 89.68 & $A$ \\
\hline 30 mM PO plus 100 ppm GA & 99.20 & a & 97.43 & $a b c$ & 90.12 & $a-d$ & 82.47 & def & 92.30 & A \\
\hline Means & 98.54 & $A$ & 90.69 & $B$ & 81.69 & C & 72.45 & D & & \\
\hline
\end{tabular}


Table 4. Influence of some postharvest treatments on visual appearance of guava fruits strain 'Etmany' under cold storage conditions during 2015 and 2016 seasons.

\begin{tabular}{|c|c|c|c|c|c|}
\hline \multirow{3}{*}{ Postharvest treatments } & \multicolumn{4}{|c|}{ Storage period (dav) } & \multirow{3}{*}{ Means } \\
\hline & $0+2$ & $7+2$ & $14+2$ & $21+2$ & \\
\hline & \multicolumn{4}{|c|}{2015 Season } & \\
\hline Distilled water (control) & $9.00 \mathrm{a}$ & 6.33 def & $4.00 \mathrm{a}$ & $2.67 \mathrm{~h}$ & $5.50 \mathrm{D}$ \\
\hline 30 mM ammonium oxalate (AO) & $9.00 \mathrm{a}$ & $8.67 a b$ & 6.33 def & $5.00 \mathrm{fq}$ & $7.25 \mathrm{C}$ \\
\hline 30 mM potassium oxalate (PO) & $9.00 \mathrm{a}$ & $9.00 \mathrm{a}$ & 7.67 bcd & 6.00 ef & $7.92 \mathrm{BC}$ \\
\hline 50 ppm qibberellic acid $\left(\mathrm{GA}_{3}\right)$ & $9.00 \mathrm{a}$ & $8.67 a b$ & 6.00 ef & $5.00 \mathrm{fq}$ & $7.17 \mathrm{C}$ \\
\hline 100 ppm GA 3 & $9.00 \mathrm{a}$ & $9.00 \mathrm{a}$ & 7.33 bcd & 6.00 ef & $7.83 \mathrm{BC}$ \\
\hline $30 \mathrm{mM}$ AO plus 50 ppm GA 3 & $9.00 \mathrm{a}$ & $9.00 \mathrm{a}$ & $8.33 a b$ & $6.67 \mathrm{c}-\mathrm{f}$ & $8.25 \mathrm{AB}$ \\
\hline $30 \mathrm{mM}$ AO plus 100 ppm GA $\mathrm{A}_{3}$ & $9.00 \mathrm{a}$ & $9.00 \mathrm{a}$ & $8.67 \mathrm{ab}$ & 7.33 b-e & $8.50 \mathrm{AB}$ \\
\hline 30 mM PO plus 50 ppm GA 3 & $9.00 \mathrm{a}$ & $9.00 \mathrm{a}$ & $8.33 a b$ & $7.00 \mathrm{c}-\mathrm{f}$ & $8.33 \mathrm{AB}$ \\
\hline $30 \mathrm{mM}$ PO plus 100 ppm GA $\mathrm{G}_{3}$ & $9.00 \mathrm{a}$ & $9.00 \mathrm{a}$ & $9.00 \mathrm{a}$ & $8.00 a b c$ & $8.75 \mathrm{~A}$ \\
\hline \multirow[t]{2}{*}{ Means } & $9.00 \mathrm{~A}$ & $8.63 \mathrm{~A}$ & $7.30 \mathrm{~B}$ & $5.96 \mathrm{C}$ & \\
\hline & \multicolumn{4}{|c|}{2016 Season } & \\
\hline Distilled water (control) & $9.00 \mathrm{a}$ & 6.00 def & $3.67 \mathrm{ah}$ & $2.00 \mathrm{~h}$ & $5.17 \mathrm{E}$ \\
\hline $30 \mathrm{mM}$ ammonium oxalate ( $\mathrm{AO}$ ) & $9.00 \mathrm{a}$ & $8.33 a b$ & 6.00 def & $5.00 \mathrm{fa}$ & $7.08 \mathrm{CD}$ \\
\hline 30 mM potassium oxalate (PO) & $9.00 \mathrm{a}$ & $9.00 \mathrm{a}$ & 7.33 a-e & 5.67 ef & $7.75 \mathrm{BC}$ \\
\hline 50 ppm qibberellic acid $\left(\mathrm{GA}_{3}\right)$ & $9.00 \mathrm{a}$ & $8.33 \mathrm{ab}$ & 5.67 ef & $5.00 \mathrm{fq}$ & $7.00 \mathrm{D}$ \\
\hline 100 ppm GA 3 & $9.00 \mathrm{a}$ & $9.00 \mathrm{a}$ & 7.33 a-e & 5.67 ef & $7.75 \mathrm{BC}$ \\
\hline 30 mM AO plus 50 ppm GA $\mathrm{G}_{3}$ & $9.00 \mathrm{a}$ & $9.00 \mathrm{a}$ & $8.00 \mathrm{abc}$ & $6.33 \mathrm{c}-\mathrm{f}$ & $8.08 \mathrm{AB}$ \\
\hline $30 \mathrm{mM}$ AO plus 100 ppm $\mathrm{GA}_{3}$ & $9.00 \mathrm{a}$ & $9.00 \mathrm{a}$ & $8.33 a b$ & 7.00 a-e & $8.33 \mathrm{AB}$ \\
\hline 30 mM PO plus 50 ppm GA 3 & $9.00 \mathrm{a}$ & $9.00 \mathrm{a}$ & $8.00 a b c$ & 6.33 b-e & $8.08 \mathrm{AB}$ \\
\hline $30 \mathrm{mM}$ PO plus 100 ppm $\mathrm{GA}_{3}$ & $9.00 \mathrm{a}$ & $9.00 \mathrm{a}$ & $9.00 \mathrm{a}$ & 7.67 a-d & $8.67 \mathrm{~A}$ \\
\hline Means & $9.00 \mathrm{~A}$ & $8.52 \mathrm{~B}$ & $7.04 \mathrm{C}$ & $5.63 \mathrm{D}$ & \\
\hline
\end{tabular}

Means followed by the same letters season are not significantly different at level $P \leq 0.05$ according to DMRT.

\section{Fruit firmness}

Fruit firmness is often the first quality character judged by the consumer and extremely important in overall product acceptance. Fruit firmness changes during 21 days of storage at $8^{\circ} \mathrm{C}$ plus two days shelf life are shown in Table 5 . The firmness values of treated guavas fruits strain 'Etmany' were significantly higher compared to the control (untreated) fruits. $30 \mathrm{mM}$ potassium or ammonium oxalate in combination with gibberellic acid at $100 \mathrm{ppm}$ caused higher firmness values than the other treatments in the two seasons.

Fruits treated with dipping in $30 \mathrm{mM}$ potassium oxalate plus gibberellic acid at $100 \mathrm{ppm}$ recorded the highest significant value of firmness $\left(6.01 \& 5.08 \mathrm{~N} / \mathrm{cm}^{2}\right)$ in the two seasons, respectively. On contrary, untreated fruits showed the lowest firmness values ( $\left.4.04 \& 3.55 \mathrm{~N} / \mathrm{cm}^{2}\right)$ in both seasons, respectively

Regarding the effect of storage period, data in Table 5 indicated that, a gradual and significant decrease in fruit firmness was observed during storage period in the two seasons.

Referring to the interaction effect, there was a significant interaction between pre-storage treatments and storage periods at $(p \leq 0.05)$ for firmness in both seasons in this study. 
Our results are in agreement with those obtained by Vicente et al. (2007) who indicated that, guava suffers a rapid loss of firmness during senescence, which contributes greatly to its short postharvest life and susceptibility to fungal contamination. The rate of fruit firmness losses may influence not only fruit quality but also the storage life. The loss in fruit firmness starts with the conversion of insoluble protopectin into water soluble pectin and also associated with dissolution of the middle lamallae that lead to an increase of pectin solubility and depolymerization of matrix polysaccharides. Hydrolytic enzyme activity such as pectin methylesterase, polygalacturonase and betagalactosidase are contributor in reduced rigidity of cell walls and an increase in activities of these enzymes leading to fruit softening (Payasi et al., 2009). Also, Reena, (2016) reported that, guava fruits treated with gibberellic acid showed higher firmness as compared to untreated.

Hence, the maintenance of higher firmness by application of potassium or ammonium oxalate and gibberellic acid especially in combination may be due to the role of these compounds in maintaining cellular organization and reducing cell wall enzyme activities. That led to slowing down the ripening process by retarding the pre climacteric respiration rate and consequently ethylene production (Zheng et al., 2007a, b; Zheng et al., 2012 and Reena, 2016).

Table 5. Influence of some postharvest treatments on firmness $\left(\mathrm{N} / \mathrm{cm}^{2}\right)$ of guava fruits strain 'Etmany' under cold storage conditions during 2015 and 2016 seasons.

\begin{tabular}{|c|c|c|c|c|c|}
\hline \multirow{3}{*}{ Postharvest treatments } & \multicolumn{4}{|c|}{ Storaqe period (dav) } & \multirow{3}{*}{ Means } \\
\hline & $0+2$ & $7+2$ & $14+2$ & $21+2$ & \\
\hline & \multicolumn{4}{|c|}{2015 Season } & \\
\hline Distilled water (control) & $6.69 a b$ & 4.37 ah & $3.18 \mathrm{Im}$ & $1.91 \mathrm{n}$ & $4.04 \mathrm{~F}$ \\
\hline $30 \mathrm{mM}$ ammonium oxalate ( $\mathrm{AO}$ ) & $6.81 \mathrm{ab}$ & 5.18 cde & $4.10 \mathrm{ij}$ & $2.52 \mathrm{Im}$ & 4.65 DE \\
\hline 30 mM potassium oxalate (PO) & $6.83 a b$ & $5.51 \mathrm{~cd}$ & 4.58 ghi & $2.87 \mathrm{kl}$ & $4.95 \mathrm{C}$ \\
\hline 50 ppm qibberellic acid $\left(\mathrm{GA}_{3}\right)$ & $6.80 \mathrm{ab}$ & 4.99 def & $3.74 \mathrm{i}$ & $2.29 \mathrm{~m}$ & $4.46 \mathrm{E}$ \\
\hline $100 \mathrm{ppm} \mathrm{GA} 3$ & $6.81 \mathrm{ab}$ & $5.45 \mathrm{~cd}$ & 4.47 hi & $2.83 \mathrm{kl}$ & $4.89 \mathrm{CD}$ \\
\hline 30 mM AO plus 50 ppm GA 3 & $6.84 a b$ & $5.87 \mathrm{~cd}$ & 4.78 fah & $3.45 \mathrm{ik}$ & $5.23 \mathrm{~B}$ \\
\hline $30 \mathrm{mM}$ AO plus 100 ppm GA 3 & 6.85 a & $6.55 \mathrm{~b}$ & 5.45 cde & $4.14 \mathrm{hi}$ & $5.75 \mathrm{~A}$ \\
\hline $30 \mathrm{mM}$ PO plus 50 ppm GA 3 & $6.84 a b$ & $6.03 \mathrm{c}$ & 5.07 efa & $3.82 \mathrm{ii}$ & $5.44 \mathrm{~B}$ \\
\hline $30 \mathrm{mM}$ PO plus 100 ppm GA & 6.87 a & $6.78 \mathrm{ab}$ & $5.72 \mathrm{~cd}$ & $4.66 \mathrm{fgh}$ & $6.01 \mathrm{~A}$ \\
\hline \multirow[t]{2}{*}{ Means } & $6.82 \mathrm{~A}$ & $5.64 \mathrm{~B}$ & $4.57 \mathrm{C}$ & $3.16 \mathrm{D}$ & \\
\hline & \multicolumn{4}{|c|}{2016 Season } & \\
\hline Distilled water (control) & $6.16 \mathrm{a}$ & 4.13 iik & 2.39 no & $1.52 \mathrm{r}$ & $3.55 \mathrm{~F}$ \\
\hline 30 mM ammonium oxalate (AO) & $6.37 \mathrm{a}$ & 4.89 def & $3.58 \mathrm{jkl}$ & $2.37 \mathrm{pq}$ & 4.30 DE \\
\hline 30 mM potassium oxalate (PO) & $6.40 \mathrm{a}$ & 5.20 cde & $4.03 \mathrm{a}-i$ & $2.71 \mathrm{op}$ & $4.59 \mathrm{C}$ \\
\hline 50 ppm qibberellic acid $\left(\mathrm{GA}_{3}\right)$ & $6.36 a$ & 4.71 e-h & $3.24 \mathrm{Im}$ & $2.16 \mathrm{qr}$ & $4.12 \mathrm{E}$ \\
\hline $100 \mathrm{ppm} \mathrm{GA} 3$ & $6.38 \mathrm{a}$ & 5.14 cde & $3.93 \mathrm{hij}$ & $2.67 \mathrm{opq}$ & $4.53 \mathrm{CD}$ \\
\hline $30 \mathrm{mM}$ AO plus 50 ppm $\mathrm{GA}_{3}$ & $6.42 \mathrm{a}$ & $5.24 \mathrm{c}$ & $4.23 \mathrm{f}-\mathrm{i}$ & $3.08 \mathrm{mn}$ & $4.74 \mathrm{BC}$ \\
\hline $30 \mathrm{mM}$ AO plus 100 ppm GA 3 & $6.45 a$ & $5.89 a b$ & 4.86 cde & $3.83 \mathrm{jkl}$ & $5.26 \mathrm{~A}$ \\
\hline 30 mM PO plus 50 ppm GA 3 & $6.43 \mathrm{a}$ & 5.40 bc & $4.51 \mathrm{efq}$ & $3.53 \mathrm{klm}$ & $4.97 \mathrm{~B}$ \\
\hline $30 \mathrm{mM}$ PO plus 100 ppm GA3 & 6.48 a & 6.11 a & $5.12 \mathrm{~cd}$ & $4.23 \mathrm{f}-\mathrm{i}$ & $5.48 \mathrm{~A}$ \\
\hline Means & $6.38 \mathrm{~A}$ & $5.19 \mathrm{~B}$ & $3.99 \mathrm{C}$ & $2.90 \mathrm{D}$ & \\
\hline
\end{tabular}

Means followed by the same letters season are not significantly different at level $P \leq 0.05$ according to DMRT. 


\section{Skin colour and lightness}

Colour is an important factor determining the appearance of fruits and consequently its quality. The perusal in Tables 6 and 7 indicated that, all pre-storage treatments especially in combination treatments significantly delayed guava fruits strain 'Etmany' skin colour development during storage compared with untreated fruits. Fruit colour, represented as hue angle value, changed from yellowish green (more than 90) to greenish yellow or yellow (less than 90). Also, these treatments significantly reduced the decreasing rate of gloss, represented as, lightness as compared to control fruits in both seasons. Thus, treated fruits still greener and glossier than untreated fruits. Guava fruits treated with $30 \mathrm{mM}$ potassium or ammonium oxalate plus gibberellic acid at $100 \mathrm{ppm}$ were superior in constricting the changing rate of skin colour and showed the highest values of hue angle and lightness than the other treatments.

Fruits treated with $30 \mathrm{mM}$ potassium oxalate plus gibberellic acid at $100 \mathrm{ppm}$ as dipping treatment gained the maximum values of hue angle (99.63 \& 94.29) and lightness (79.62 \& 80.67) compared with the other treatments. On the other hand, control fruits recorded the minimum values of both hue angle $(89.07 \& 84.24)$ and lightness (70.56 \& 70.39) in the two seasons, respectively.

As the effect of storage period, skin colour of guava fruits changed directly from yellowish green to yellow during storage at $8^{\circ} \mathrm{C}$ plus two days at ambient temperature $\left(18-23^{\circ} \mathrm{C}\right)$. On the other side, lightness values gradually and significantly decreased with prolonging storage period during the two seasons in this experiment. The interaction effect between pre-storage treatments and storage periods showed significant difference interactions at $(p \leq 0.05)$ for hue angle and lightness during the two seasons in this research. Decreasing in hue angle values with the advancement of storage period probably was due to the disappearance of the green colour in fruits because of chlorophyll degradation and the concomitant unmasking or synthesis of carotenoids which gives the fruits a yellow color (Shewfelt, 1993). Gibberellic acid reported to extend the storage life of citrus fruits by delaying chlorophyll degradation and the fruit senescence (Mohamed et al., 2016a). Moreover, pre-storage application with gibberellic acid delayed skin colour development during storage by retards ripening process of mangoes (Mohamed et al., 2016b) and bananas (Duguma et al., 2014). Furthermore, oxalic acid treatment 
Table 6. Influence of some postharvest treatments on color represented as hue angle of guava fruits strain 'Etmany' under cold storage conditions during 2015 and 2016 seasons.

\begin{tabular}{|c|c|c|c|c|c|c|c|c|c|}
\hline \multirow{3}{*}{ Postharvest treatments } & \multicolumn{7}{|c|}{ Storage period (day) } & \multirow{3}{*}{\multicolumn{2}{|c|}{ Means }} \\
\hline & \multicolumn{2}{|c|}{$0+2$} & \multicolumn{2}{|c|}{$7+2$} & \multicolumn{2}{|c|}{$14+2$} & $21+2$ & & \\
\hline & \multicolumn{7}{|c|}{2015 Season } & & \\
\hline Distilled water (control) & 108.49 & $a$ & 90.04 & fgh & 82.43 & $\mathrm{k}$ & 75.31 I & 89.07 & \\
\hline $30 \mathrm{mM}$ ammonium oxalate (AO) & 110.36 & a & 97.51 & $\mathrm{~cd}$ & 86.25 & ij & $84.94 j$ & 94.76 & $\mathrm{~F}$ \\
\hline 30 mM potassium oxalate $(\mathrm{PO})$ & 110.66 & a & 98.85 & bc & 88.78 & ghi & $85.86 \mathrm{ij}$ & 96.04 & DEF \\
\hline 50 ppm gibberellic acid $\left(\mathrm{GA}_{3}\right)$ & 110.32 & a & 98.73 & $\mathrm{bc}$ & 87.24 & hij & $85.61 j$ & 95.48 & $\mathrm{EF}$ \\
\hline $100 \mathrm{ppm} \mathrm{GA}$ & 110.72 & a & 99.06 & bc & 88.83 & ghi & $86.19 \mathrm{ij}$ & 96.20 & $\mathrm{DE}$ \\
\hline $30 \mathrm{mM}$ AO plus 50 ppm $\mathrm{GA}_{3}$ & 110.88 & a & 99.20 & bc & 90.04 & fgh & 87.69 hij & 96.95 & $C D$ \\
\hline $30 \mathrm{mM}$ AO plus 100 ppm $\mathrm{GA}_{3}$ & 111.10 & a & 100.89 & $b$ & 93.77 & $\mathrm{e}$ & $89.54 \mathrm{gh}$ & 98.82 & $A B$ \\
\hline $30 \mathrm{mM}$ PO plus 50 ppm GA & 110.95 & a & 100.52 & $\mathrm{~b}$ & 92.66 & ef & 88.61 ghi & 98.18 & $\mathrm{BC}$ \\
\hline $30 \mathrm{mM}$ PO plus $100 \mathrm{ppm} \mathrm{GA}$ & 111.47 & $\mathrm{a}$ & 101.42 & $b$ & 94.93 & de & $90.71 \mathrm{fg}$ & 99.63 & A \\
\hline \multirow[t]{2}{*}{ Means } & 110.55 & $\mathrm{~A}$ & 98.47 & $\mathrm{~B}$ & 89.44 & $\mathrm{C}$ & $86.05 \mathrm{D}$ & & \\
\hline & \multicolumn{7}{|c|}{2016 Season } & & \\
\hline Distilled water (control) & 102.67 & b & 85.21 & fgh & 76.34 & I & $72.75 \mathrm{~m}$ & 84.24 & \\
\hline $30 \mathrm{mM}$ ammonium oxalate $(\mathrm{AO})$ & 104.44 & $a b$ & 92.28 & $d$ & 81.62 & $\mathrm{ijk}$ & $80.38 \mathrm{kl}$ & 89.68 & $\mathrm{~F}$ \\
\hline 30 mM potassium oxalate (PO) & 104.72 & $a b$ & 93.54 & $\mathrm{~cd}$ & 84.02 & ghi & $81.25 \mathrm{jk}$ & 90.89 & DEF \\
\hline 50 ppm gibberellic acid $\left(\mathrm{GA}_{3}\right)$ & 104.40 & $a b$ & 93.43 & $\mathrm{~cd}$ & 82.56 & $\mathrm{~h}-\mathrm{k}$ & $81.02 \mathrm{k}$ & 90.35 & $\mathrm{EF}$ \\
\hline $100 \mathrm{ppm} \mathrm{GA}$ & 104.84 & $a b$ & 93.75 & $\mathrm{~cd}$ & 84.07 & ghi & $81.56 \mathrm{ijk}$ & 91.06 & $\mathrm{DE}$ \\
\hline $30 \mathrm{mM}$ AO plus 50 ppm $\mathrm{GA}_{3}$ & 104.93 & $a b$ & 93.88 & $\mathrm{~cd}$ & 85.21 & fgh & 82.99 h-k & 91.75 & $\mathrm{CD}$ \\
\hline $30 \mathrm{mM}$ AO plus $100 \mathrm{ppm} \mathrm{GA}_{3}$ & 105.14 & $a b$ & 95.47 & $\mathrm{c}$ & 88.74 & e & $84.74 \mathrm{gh}$ & 93.52 & $A B$ \\
\hline $30 \mathrm{mM}$ PO plus 50 ppm $\mathrm{GA}_{3}$ & 105.00 & $a b$ & 95.12 & c & 87.69 & ef & $83.86 \mathrm{~g}-\mathrm{j}$ & 92.92 & $\mathrm{BC}$ \\
\hline $30 \mathrm{mM}$ PO plus 100 ppm GA 3 & 105.49 & a & 95.98 & c & 89.84 & $\mathrm{e}$ & $85.85 \mathrm{fg}$ & 94.29 & $A$ \\
\hline Means & 104.62 & $A$ & 93.19 & B & 84.45 & $\mathrm{C}$ & $81.60 \mathrm{D}$ & & \\
\hline
\end{tabular}

Means followed by the same letters season are not significantly different at level $\mathrm{P} \leq 0.05$ according to DMRT.

Table 7. Influence of some postharvest treatments on lightness of guava fruits strain 'Etmany' under cold storage conditions during 2015 and 2016 seasons.

\begin{tabular}{|c|c|c|c|c|c|}
\hline \multirow{3}{*}{ Postharvest treatments } & \multicolumn{4}{|c|}{ Storage period (day) } & \multirow{3}{*}{ Means } \\
\hline & $0+2$ & $7+2$ & $14+2$ & $21+2$ & \\
\hline & \multicolumn{4}{|c|}{2015 Season } & \\
\hline Distilled water (control) & $81.43 \mathrm{ab}$ & $74.03 \mathrm{gh}$ & $67.10 \mathrm{k}$ & $59.66 \quad$ & $70.56 E$ \\
\hline $30 \mathrm{mM}$ ammonium oxalate (AO) & 82.79 a & $78.43 \mathrm{c}-\mathrm{f}$ & $72.23 \mathrm{gh}$ & $67.01 \mathrm{j}$ & $75.12 \mathrm{D}$ \\
\hline $30 \mathrm{mM}$ potassium oxalate (PO) & $83.01 \mathrm{a}$ & 79.85 b-e & $74.32 \mathrm{fg}$ & $69.08 \mathrm{hij}$ & $76.56 \mathrm{CD}$ \\
\hline 50 ppm gibberellic acid $\left(\mathrm{GA}_{3}\right)$ & 82.76 a & $78.56 \mathrm{c}-f$ & $72.88 \mathrm{gh}$ & $67.69 \mathrm{ij}$ & $75.47 \mathrm{D}$ \\
\hline $100 \mathrm{ppm} \mathrm{GA} 3$ & $82.90 \mathrm{a}$ & 79.68 b-e & $74.65 \mathrm{fg}$ & $68.65 \mathrm{hij}$ & $76.47 \mathrm{CD}$ \\
\hline $30 \mathrm{mM}$ AO plus 50 ppm $\mathrm{GA}_{3}$ & $83.17 \mathrm{a}$ & 79.61 b-e & $75.98 \mathrm{fg}$ & $72.14 \mathrm{ghi}$ & $77.72 \mathrm{BC}$ \\
\hline $30 \mathrm{mM}$ AO plus 100 ppm $\mathrm{GA}_{3}$ & $83.35 \mathrm{a}$ & 80.19 bcd & 77.34 efg & $74.25 \mathrm{fg}$ & $78.78 \mathrm{AB}$ \\
\hline $30 \mathrm{mM}$ PO plus 50 ppm $\mathrm{GA}_{3}$ & $83.23 \mathrm{a}$ & 80.05 bcd & $76.83 \mathrm{fg}$ & $72.84 \mathrm{gh}$ & $78.24 \mathrm{AB}$ \\
\hline $30 \mathrm{mM}$ PO plus 100 ppm GA & $83.63 \mathrm{a}$ & $81.60 \mathrm{abc}$ & 78.47 def & $74.77 \mathrm{fg}$ & $79.62 \mathrm{~A}$ \\
\hline \multirow[t]{2}{*}{ Means } & $82.92 \mathrm{~A}$ & $79.11 \mathrm{~B}$ & $74.42 \mathrm{C}$ & $69.57 \mathrm{D}$ & \\
\hline & \multicolumn{4}{|c|}{2016 Season } & \\
\hline Distilled water (control) & 83.00 a-f & $74.53 \mathrm{klm}$ & $65.46 n$ & 58.580 & $70.39 \mathrm{E}$ \\
\hline $30 \mathrm{mM}$ ammonium oxalate (AO) & 84.39 a-e & $78.95 \mathrm{f}-\mathrm{i}$ & $74.19 \mathrm{~m}$ & $70.36 \mathrm{n}$ & $76.97 \mathrm{D}$ \\
\hline 30 mM potassium oxalate (PO) & 84.61 abc & $80.39 \mathrm{c}-\mathrm{h}$ & $76.32 \mathrm{j}-\mathrm{m}$ & $72.47 \mathrm{n}$ & $78.45 \mathrm{BCD}$ \\
\hline 50 ppm gibberellic acid $\left(\mathrm{GA}_{3}\right)$ & 84.36 a-e & $79.08 \mathrm{f}-\mathrm{i}$ & $74.85 \mathrm{Im}$ & $71.05 \mathrm{n}$ & 77.34 CD \\
\hline 100 ppm GA 3 & 84.62 a-d & 80.21 d-h & $76.66 j-m$ & $72.03 \mathrm{n}$ & $78.38 \mathrm{BCD}$ \\
\hline $30 \mathrm{mM}$ AO plus 50 ppm $\mathrm{GA}_{3}$ & $84.78 a b$ & 80.14 e-h & 75.98 i-I & $74.06 \mathrm{~m}$ & 78.74 BC \\
\hline $30 \mathrm{mM}$ AO plus 100 ppm GA & $84.96 a b$ & 80.74 b-g & $77.36 \mathrm{~g}-\mathrm{j}$ & $76.21 \mathrm{j}-\mathrm{m}$ & $79.82 \mathrm{AB}$ \\
\hline $30 \mathrm{mM}$ PO plus 50 ppm GA 3 & $84.84 a b$ & 80.60 b-h & 76.84 h-k & $74.77 \mathrm{Im}$ & $79.26 \mathrm{AB}$ \\
\hline $30 \mathrm{mM}$ PO plus 100 ppm GA 3 & 85.24 a & 82.18 a-f & $78.52 \mathrm{f}-\mathrm{i}$ & $76.74 \mathrm{j}-\mathrm{m}$ & $80.67 \mathrm{~A}$ \\
\hline Means & $84.53 \mathrm{~A}$ & 79.65 B & $75.13 \mathrm{C}$ & $71.81 \mathrm{D}$ & \\
\hline
\end{tabular}

Means followed by the same letters season are not significantly different at level $\mathrm{P} \leq 0.05$ according to DMRT. 
increased hue angle value and constricted the variability in skin colour during storage of oranges (Mohamed et al., 2016a) and bananas (Huang et al., 2013).

In this research, the higher colour retention of guavas by retaining the highest hue angle value with potassium or ammonium oxalate and gibberellic acid especially in combination treatments as compared with control (untreated) fruits may be due to the slower change of chlorophyll into carotenoids. Moreover, all studied treatments especially in combination treatments increased the gloss of guavas represented as lightness than control treatment, which might be due to its anti senescence action (Mohamed et al., 2016).

\subsubsection{Chemical Characteristics:}

Vitamin C contents, Total Soluble Solids (TSS), Total Acidity (TA) and TSS / TA ratio

Data tabulated in Tables 8, 9, 10 and 11 clearly indicated that all postharvest treatments of guava fruits strain 'Etmany' significantly reduced the deterioration rate in vitamin C content and TA than control (untreated) fruits. However, the decreases in vitamin $\mathrm{C}$ content and TA during storage were more rapid in control fruits. In addition, treated guavas significantly slowed an increase in TSS content and TSS/TA ratio as compared to control (untreated) guavas during the two seasons under this study. Moreover, fruits treated with $30 \mathrm{mM}$ potassium or ammonium oxalate plus gibberellic acid at $100 \mathrm{ppm}$ were significantly had the highest values of Vitamin $\mathrm{C}$ and titratable acidity as well as the lowest values of TSS and TSS/TA ratio in both seasons.

In addition, fruits treated with $30 \mathrm{mM}$ potassium oxalate in combination with gibberellic acid at $100 \mathrm{ppm}$ as a dipping treatment had the highest values of vitamin $\mathrm{C}$ (100.08 \& $96.79 \mathrm{mg} / 100 \mathrm{ml}$ juice) and TA (0.82 \& 0.74), and the lowest values of TSS ( $9.15 \& 9.39{ }^{\circ}$ Brix) and TSS/TA ratio (11.29 \& 12.71) in the two seasons, respectively. On contrary, control fruits recorded the lowest vitamin C content (83.65\& 80.10 $\mathrm{mg} / 100 \mathrm{ml}$ juice) and TA (0.59\& 0.52), while recorded the highest TSS (9.75\& 10.00 ${ }^{\circ}$ Brix) and TSS/TA ratio (18.45 \& 22.05) in the first and second seasons, respectively.

As for the effect of storage time, declining trend in vitamin C content and TA was observed with advancing the storage period at $8^{\circ} \mathrm{C}$ plus two days at ambient temperature $\left(18-23^{\circ} \mathrm{C}\right)$ in the two seasons. On the other side, TSS content significantly increased up to 14 days of cold storage plus 2 days shelf life followed by a slight and insignificant decrease until end of the experiment, while rising trend in TSS/TA ratio was recorded with the advancement of storage period in both seasons.

Data also declared that, there was a significant interaction among pre-storage treatments and storage periods at $(p \leq 0.05)$ for these attributes in both seasons in the current study. The loss of ascorbic acid content of guavas during storage time might 
be due to rapid conversion of L-ascorbic acid into dehydroascorbic acid in the presence of oxidizing enzymes like ascorbic acid oxidase and ascorbate peroxidase (Mapson, 1970).

Increased vitamin C content in Guavas strain 'Etmany' treated with potassium or ammonium oxalate and gibberellic acid especially in combination may be ascribed to reducing respiration of fruits or oxidation of ascorbic acid content by suppression of ascorbate oxidase activity. These results are in line with the findings of (Reena, 2016) in guavas (Zheng et al., 2007 and Mohamed et al., 2016b) in mangoes, (Duguma et al., 2014) in bananas and (Mohamed et al., 2016a) in Valencia oranges, they reported that vitamin $\mathrm{C}$ was retained by using gibberellic acid or oxalic acid treatment during storage. Moreover, they demonstrated that vitamin $\mathrm{C}$ decreased with the elongation of storage time.

A progressive decline in TA of guavas during storage might be due to utilization of organic acids in respiration process and conversion of acids into salts and sugars by the enzymes (Kays, 1991). On the other hand, the increasing trend in TSS during storage time may possibly be due to hydrolysis of starch into sugars, and due to the

Table 8. Influence of some postharvest treatments on vitamin C content as $\mathrm{mg} / 100$ $\mathrm{ml}$ of the juice of guava fruits strain 'Etmany' under cold storage conditions during 2015 and 2016 seasons.

\begin{tabular}{|c|c|c|c|c|c|c|c|c|}
\hline \multirow{3}{*}{ Postharvest treatments } & \multicolumn{6}{|c|}{ Storage period (day) } & & \\
\hline & \multicolumn{2}{|l|}{$0+2$} & \multicolumn{2}{|c|}{$7+2$} & $14+2$ & $21+2$ & \multicolumn{2}{|c|}{ Means } \\
\hline & \multicolumn{6}{|c|}{2015 Season } & & \\
\hline Distilled water (control) & 111.28 & a & 86.67 & $f-j$ & 73.33 I & $63.33 \mathrm{~m}$ & 83.65 & D \\
\hline $30 \mathrm{mM}$ ammonium oxalate (AO) & 112.82 & a & 93.33 & $b-g$ & $84.76 \mathrm{~g}-\mathrm{k}$ & $77.22 \mathrm{kl}$ & 92.03 & C \\
\hline $30 \mathrm{mM}$ potassium oxalate $(\mathrm{PO})$ & 113.85 & a & 96.36 & $b-f$ & $89.52 \mathrm{c}-\mathrm{g}$ & $80.00 \mathrm{~h}-\mathrm{k}$ & 94.93 & BC \\
\hline 50 ppm gibberellic acid $\left(\mathrm{GA}_{3}\right)$ & 112.82 & a & 93.94 & $b-g$ & $86.19 \mathrm{f}-\mathrm{j}$ & 77.78 jkl & 92.68 & C \\
\hline $100 \mathrm{ppm} \mathrm{GA}$ & 113.33 & a & 95.15 & $b-f$ & $87.62 \mathrm{~d}-\mathrm{h}$ & $78.89 \mathrm{ijk}$ & 93.75 & C \\
\hline $30 \mathrm{mM} \mathrm{AO}$ plus $50 \mathrm{ppm} \mathrm{GA}_{3}$ & 113.85 & a & 99.39 & bc & $92.86 \mathrm{~b}-\mathrm{f}$ & $86.11 \mathrm{f}-\mathrm{j}$ & 98.05 & $A B$ \\
\hline $30 \mathrm{mM}$ AO plus 100 ppm GA 3 & 114.36 & a & 101.21 & $\mathrm{~b}$ & $94.29 \mathrm{bcd}$ & $87.78 \mathrm{~d}-\mathrm{h}$ & 99.41 & $A$ \\
\hline $30 \mathrm{mM}$ PO plus 50 ppm GA 3 & 114.35 & a & 100.00 & $\mathrm{bc}$ & 93.33 b-e & 86.67 e-i & 98.59 & A \\
\hline $30 \mathrm{mM}$ PO plus $100 \mathrm{ppm} \mathrm{GA}$ & 114.36 & a & 101.82 & $\mathrm{~b}$ & 95.24 bc & $88.89 \mathrm{c}-\mathrm{g}$ & 100.08 & A \\
\hline \multirow[t]{2}{*}{ Means } & 113.45 & $\mathrm{~A}$ & 96.43 & $\mathrm{~B}$ & $88.57 \mathrm{C}$ & $80.74 \mathrm{D}$ & & \\
\hline & \multicolumn{6}{|c|}{2016 Season } & & \\
\hline Distilled water (control) & 106.00 & a & 83.89 & $h-k$ & $70.00 \mathrm{~m}$ & $60.51 \mathrm{n}$ & 80.10 & $\mathrm{D}$ \\
\hline $30 \mathrm{mM}$ ammonium oxalate $(\mathrm{AO})$ & 107.80 & a & 88.89 & $d-i$ & $82.22 \mathrm{jkl}$ & $75.90 \mathrm{~m}$ & 88.70 & $\mathrm{C}$ \\
\hline $30 \mathrm{mM}$ potassium oxalate $(\mathrm{PO})$ & 108.08 & a & 91.67 & b-e & 87.78 e-j & $78.97 \mathrm{klm}$ & 91.62 & $\mathrm{BC}$ \\
\hline 50 ppm gibberellic acid $\left(\mathrm{GA}_{3}\right)$ & 107.76 & a & 89.44 & $c-h$ & $83.89 i j \mathrm{k}$ & $76.41 \mathrm{~m}$ & 89.38 & $\mathrm{C}$ \\
\hline $100 \mathrm{ppm} \mathrm{GA}$ & 107.95 & a & 90.56 & $b-f$ & $85.56 \mathrm{~g}-\mathrm{j}$ & $77.44 \mathrm{Im}$ & 90.37 & C \\
\hline $30 \mathrm{mM}$ AO plus 50 ppm GA & 108.31 & a & 94.44 & bcd & $91.67 \mathrm{~d}-\mathrm{i}$ & $84.10 \mathrm{ijk}$ & 94.63 & $A B$ \\
\hline $30 \mathrm{mM} \mathrm{AO}$ plus $100 \mathrm{ppm} \mathrm{GA}_{3}$ & 108.53 & a & 96.11 & bc & $93.33 \mathrm{c}-\mathrm{g}$ & $86.15 \mathrm{~g}-\mathrm{j}$ & 96.03 & $A$ \\
\hline $30 \mathrm{mM}$ PO plus 50 ppm $\mathrm{GA}_{3}$ & 108.38 & a & 95.00 & bcd & $92.22 \mathrm{~d}-\mathrm{i}$ & $85.13 \mathrm{~h}-\mathrm{k}$ & 95.18 & $A B$ \\
\hline $30 \mathrm{mM}$ PO plus $100 \mathrm{ppm} \mathrm{GA}_{3}$ & 108.89 & a & 96.67 & $b$ & 94.44 b-f & $87.18 \mathrm{f}-\mathrm{j}$ & 96.79 & A \\
\hline Means & 107.97 & A & 91.85 & B & $86.79 \mathrm{C}$ & $79.09 \mathrm{D}$ & & \\
\hline
\end{tabular}

Means followed by the same letters season are not significantly different at level $\mathrm{P} \leq 0.05$ according to DMRT. 
Table 9. Influence of some postharvest treatments on TSS content of guava fruits strain 'Etmany' under cold storage conditions during 2015 and 2016 seasons.

\begin{tabular}{|c|c|c|c|c|c|c|c|}
\hline \multirow{3}{*}{ Postharvest treatments } & \multicolumn{5}{|c|}{ Storage period (day) } & \multirow{3}{*}{\multicolumn{2}{|c|}{ Means }} \\
\hline & $0+2$ & & +2 & $14+2$ & $21+2$ & & \\
\hline & \multicolumn{5}{|c|}{2015 Season } & & \\
\hline Distilled water (control) & $8.07 \mathrm{I}$ & 10.80 & $a b c$ & 10.53 b-e & 9.60 ghi & 9.75 & $A B$ \\
\hline $30 \mathrm{mM}$ ammonium oxalate $(\mathrm{AO})$ & 8.051 & 9.67 & $\mathrm{ij}$ & $11.00 \mathrm{ab}$ & 10.60 a-e & 9.83 & $A$ \\
\hline $30 \mathrm{mM}$ potassium oxalate $(\mathrm{PO})$ & $8.05 \mathrm{I}$ & 9.60 & j & $10.93 \mathrm{abc}$ & 10.67 a-e & 9.81 & A \\
\hline 50 ppm gibberellic acid $\left(\mathrm{GA}_{3}\right)$ & 8.061 & 9.73 & hij & $11.07 \mathrm{a}$ & 10.53 b-e & 9.85 & A \\
\hline $100 \mathrm{ppm} \mathrm{GA}_{3}$ & 8.051 & 9.53 & $\mathrm{jk}$ & $10.90 \mathrm{abc}$ & 10.73 a-d & 9.80 & A \\
\hline $30 \mathrm{mM}$ AO plus 50 ppm $\mathrm{GA}_{3}$ & 8.041 & 9.27 & j & 10.40 b-e & $10.73 \mathrm{abc}$ & 9.61 & $A B$ \\
\hline $30 \mathrm{mM} \mathrm{AO}$ plus 100 ppm $\mathrm{GA}_{3}$ & 8.031 & 9.07 & $\mathrm{jk}$ & 10.00 def & 10.33 a-e & 9.36 & $C D$ \\
\hline $30 \mathrm{mM}$ PO plus 50 ppm $\mathrm{GA}_{3}$ & 8.031 & 9.20 & $\mathrm{jk}$ & 10.33 cde & 10.60 a-d & 9.54 & $\mathrm{BC}$ \\
\hline $30 \mathrm{mM}$ PO plus 100 ppm GA & $8.02 ।$ & 8.87 & $\mathrm{k}$ & $9.67 \mathrm{fgh}$ & 10.07 efg & 9.15 & $\mathrm{D}$ \\
\hline \multirow{2}{*}{ Means } & $8.04 \mathrm{C}$ & 9.53 & $\mathrm{~B}$ & $10.54 \mathrm{~A}$ & $10.43 \mathrm{~A}$ & & \\
\hline & \multicolumn{5}{|c|}{2016 Season } & & \\
\hline Distilled water (control) & $8.33 \mathrm{j}$ & 11.07 & $a b c$ & 10.67 a-e & $9.93 \mathrm{fgh}$ & 10.00 & A \\
\hline $30 \mathrm{mM}$ ammonium oxalate $(\mathrm{AO})$ & $8.27 \mathrm{j}$ & 9.53 & $\mathrm{fg}$ & $11.13 \mathrm{a}$ & $10.73 \mathrm{a}-\mathrm{d}$ & 9.92 & $A B$ \\
\hline $30 \mathrm{mM}$ potassium oxalate $(\mathrm{PO})$ & $8.26 \mathrm{j}$ & 9.47 & fgh & $11.00 \mathrm{ab}$ & $10.80 \mathrm{abc}$ & 9.88 & $A B$ \\
\hline 50 ppm gibberellic acid $\left(\mathrm{GA}_{3}\right)$ & $8.27 \mathrm{j}$ & 9.60 & $\mathrm{fg}$ & $11.20 \mathrm{a}$ & 10.67 a-e & 9.93 & $A B$ \\
\hline $100 \mathrm{ppm} \mathrm{GA}_{3}$ & $8.25 \mathrm{j}$ & 9.40 & fgh & $11.07 \mathrm{ab}$ & $10.87 \mathrm{abc}$ & 9.90 & $A B$ \\
\hline $30 \mathrm{mM}$ AO plus 50 ppm $\mathrm{GA}_{3}$ & $8.24 j$ & 9.47 & ghi & 10.67 b-e & $10.93 a b c$ & 9.83 & $A B C$ \\
\hline $30 \mathrm{mM} \mathrm{AO}$ plus 100 ppm $\mathrm{GA}_{3}$ & $8.23 \mathrm{j}$ & 9.20 & hi & 10.40 ef & 10.73 cde & 9.64 & $\mathrm{C}$ \\
\hline $30 \mathrm{mM}$ PO plus $50 \mathrm{ppm} \mathrm{GA_{3 }}$ & $8.24 j$ & 9.33 & ghi & 10.60 cde & 10.87 a-d & 9.76 & $\mathrm{BC}$ \\
\hline $30 \mathrm{mM}$ PO plus 100 ppm GA 3 & $8.22 \mathrm{j}$ & 9.00 & i & $10.00 \mathrm{fg}$ & 10.33 def & 9.39 & $\mathrm{D}$ \\
\hline Means & $8.26 \mathrm{C}$ & 9.56 & B & $10.75 \mathrm{~A}$ & $10.65 \mathrm{~A}$ & & \\
\hline
\end{tabular}

Means followed by the same letters season are not significantly different at level $\mathrm{P} \leq 0.05$ according to DMRT.

Table 10. Influence of some postharvest treatments on TA ( $\mathrm{g}$ citric acid/100 ml juice) of guava fruits strain 'Etmany' under cold storage conditions during 2015 and 2016 seasons.

\begin{tabular}{|c|c|c|c|c|c|}
\hline \multirow{3}{*}{ Postharvest treatments } & \multicolumn{4}{|c|}{ Storage period (day) } & \multirow{3}{*}{ Means } \\
\hline & $0+2$ & $7+2$ & $14+2$ & $21+2$ & \\
\hline & \multicolumn{4}{|c|}{2015 Season } & \\
\hline Distilled water (control) & 0.82 a-d & $0.63 \mathrm{i}-\mathrm{I}$ & $0.51 \mathrm{~m}$ & $0.40 n$ & $0.59 \mathrm{C}$ \\
\hline $30 \mathrm{mM}$ ammonium oxalate $(\mathrm{AO})$ & $0.84 a b c$ & $0.69 f-i$ & $0.59 \mathrm{i}-\mathrm{I}$ & $0.51 \mathrm{Im}$ & $0.66 \mathrm{~B}$ \\
\hline 30 mM potassium oxalate $(P O)$ & $0.87 \mathrm{abc}$ & 0.71 e-h & $0.62 \mathrm{~h}-\mathrm{k}$ & $0.57 \mathrm{j}-\mathrm{m}$ & 0.69 B \\
\hline 50 ppm gibberellic acid $\left(\mathrm{GA}_{3}\right)$ & $0.84 a b c$ & $0.67 \mathrm{~g}-\mathrm{j}$ & $0.57 \mathrm{j}-\mathrm{m}$ & $0.49 \mathrm{Im}$ & $0.64 \mathrm{~B}$ \\
\hline $100 \mathrm{ppm} \mathrm{GA}$ & $0.87 \mathrm{ab}$ & 0.69 e-h & 0.62 h-k & $0.55 \mathrm{klm}$ & 0.68 B \\
\hline $30 \mathrm{mM}$ AO plus 50 ppm $\mathrm{GA}_{3}$ & $0.87 a b$ & $0.83 a b c$ & $0.76 \mathrm{c}-\mathrm{f}$ & $0.68 \mathrm{e}-\mathrm{h}$ & $0.78 \mathrm{~A}$ \\
\hline $30 \mathrm{mM}$ AO plus $100 \mathrm{ppm} \mathrm{GA_{3 }}$ & $0.89 a$ & $0.85 \mathrm{a}-\mathrm{d}$ & 0.79 a-d & $0.72 \mathrm{c}-\mathrm{f}$ & $0.81 \mathrm{~A}$ \\
\hline $30 \mathrm{mM}$ PO plus 50 ppm GA & $0.89 a b$ & $0.83 a b c$ & 0.76 b-e & $0.70 \mathrm{~d}-\mathrm{g}$ & $0.79 \mathrm{~A}$ \\
\hline $30 \mathrm{mM}$ PO plus $100 \mathrm{ppm} \mathrm{GA_{3 }}$ & 0.89 a & $0.85 a b$ & $0.81 \mathrm{abc}$ & 0.74 b-e & $0.82 \mathrm{~A}$ \\
\hline \multirow[t]{2}{*}{ Means } & $0.86 \mathrm{~A}$ & $0.75 \mathrm{~B}$ & $0.67 \mathrm{C}$ & $0.60 \mathrm{D}$ & \\
\hline & \multicolumn{4}{|c|}{2016 Season } & \\
\hline Distilled water (control) & 0.73 a-d & $0.54 \mathrm{~g}-1$ & $0.45 \mathrm{Imn}$ & $0.36 n$ & $0.52 \mathrm{D}$ \\
\hline $30 \mathrm{mM}$ ammonium oxalate (AO) & $0.75 a b c$ & 0.60 e-j & $0.54 \mathrm{i}-\mathrm{m}$ & $0.47 \mathrm{Imn}$ & $0.59 \mathrm{C}$ \\
\hline 30 mM potassium oxalate $(P O)$ & $0.75 a b$ & $0.63 \mathrm{~d}-\mathrm{i}$ & $0.56 \mathrm{~h}-\mathrm{I}$ & $0.51 \mathrm{j}-\mathrm{m}$ & $0.61 \mathrm{C}$ \\
\hline 50 ppm gibberellic acid $\left(\mathrm{GA}_{3}\right)$ & $0.75 a b c$ & $0.58 \mathrm{f}-\mathrm{k}$ & $0.51 \mathrm{j}-\mathrm{m}$ & $0.47 \mathrm{mn}$ & $0.58 \mathrm{C}$ \\
\hline $100 \mathrm{ppm} \mathrm{GA}$ & $0.77 \mathrm{ab}$ & 0.63 e-j & 0.56 h-I & $0.49 \mathrm{klm}$ & $0.61 \mathrm{C}$ \\
\hline $30 \mathrm{mM}$ AO plus 50 ppm $\mathrm{GA}_{3}$ & $0.77 a b$ & 0.74 a-d & 0.67 a-f & $0.63 \mathrm{f}-\mathrm{j}$ & $0.70 \mathrm{~B}$ \\
\hline $30 \mathrm{mM} \mathrm{AO}$ plus $100 \mathrm{ppm} \mathrm{GA_{3 }}$ & 0.79 a & $0.71 \mathrm{ab}$ & 0.71 a-f & $0.67 \mathrm{c}-\mathrm{h}$ & $0.72 A B$ \\
\hline $30 \mathrm{mM}$ PO plus 50 ppm GA & $0.77 \mathrm{a}$ & 0.74 a-d & 0.69 a-f & 0.65 e-i & $0.71 A B$ \\
\hline $30 \mathrm{mM}$ PO plus 100 ppm GA & 0.79 a & $0.76 \mathrm{ab}$ & 0.74 a-e & $0.69 \mathrm{~b}-\mathrm{g}$ & $0.74 \mathrm{~A}$ \\
\hline Means & $0.76 \mathrm{~A}$ & $0.66 \mathrm{~B}$ & $0.60 \mathrm{C}$ & $0.55 \mathrm{D}$ & \\
\hline
\end{tabular}


Table 11. Influence of some postharvest treatments on TSS / TA ratio of guava fruits strain 'Etmany' under cold storage conditions during 2015 and 2016 seasons.

\begin{tabular}{|c|c|c|c|c|c|c|}
\hline \multirow{3}{*}{ Postharvest treatments } & \multicolumn{5}{|c|}{ Storage period (day) } & \multirow{3}{*}{ Means } \\
\hline & $0+$ & 2 & $7+2$ & $14+2$ & $21+2$ & \\
\hline & \multicolumn{5}{|c|}{2015 Season } & \\
\hline Distilled water (control) & 9.85 & ij & 17.32 bc & $20.95 \mathrm{~b}$ & $25.66 a$ & $18.45 \mathrm{~A}$ \\
\hline $30 \mathrm{mM}$ ammonium oxalate $(\mathrm{AO})$ & 9.57 & j & $13.99 \mathrm{efg}$ & $18.56 \mathrm{bc}$ & 21.02 bc & $15.79 \mathrm{~B}$ \\
\hline 30 mM potassium oxalate (PO) & 9.30 & j & 13.53 e-h & $18.11 \mathrm{~cd}$ & 18.80 bc & $14.94 \mathrm{~B}$ \\
\hline 50 ppm gibberellic acid $\left(\mathrm{GA}_{3}\right)$ & 9.58 & j & 14.53 ef & $19.32 \mathrm{bc}$ & $21.70 \mathrm{~b}$ & $16.28 \mathrm{~B}$ \\
\hline $100 \mathrm{ppm} \mathrm{GA}_{3}$ & 9.33 & j & 13.98 e-h & $17.98 \mathrm{~cd}$ & $19.52 \mathrm{bc}$ & $15.20 \mathrm{~B}$ \\
\hline $30 \mathrm{mM}$ AO plus 50 ppm $\mathrm{GA}_{3}$ & 9.29 & j & $11.23 \mathrm{~g}-\mathrm{j}$ & $13.68 \mathrm{efg}$ & $15.84 \mathrm{de}$ & $12.51 \mathrm{C}$ \\
\hline $30 \mathrm{mM}$ AO plus 100 ppm GA 3 & 9.05 & j & $10.85 \mathrm{~g}-\mathrm{j}$ & $12.75 \mathrm{e}-\mathrm{i}$ & 14.45 efg & $11.78 \mathrm{C}$ \\
\hline $30 \mathrm{mM}$ PO plus 50 ppm $\mathrm{GA}_{3}$ & 9.06 & j & $11.15 \mathrm{~g}-\mathrm{j}$ & 13.57 e-h & 15.21 ef & $12.25 \mathrm{C}$ \\
\hline $30 \mathrm{mM}$ PO plus 100 ppm GA 3 & 9.04 & j & $10.54 \mathrm{hij}$ & $12.02 \mathrm{f}-\mathrm{j}$ & 13.57 e-h & $11.29 \mathrm{C}$ \\
\hline \multirow[t]{2}{*}{ Means } & 9.34 & D & $13.02 \mathrm{C}$ & $16.33 \mathrm{~B}$ & $18.42 \mathrm{~A}$ & \\
\hline & \multicolumn{5}{|c|}{2016 Season } & \\
\hline Distilled water (control) & 11.50 & $\mathrm{kl}$ & $20.85 \mathrm{c}-\mathrm{g}$ & 24.02 bc & 31.82 a & $22.05 \mathrm{~A}$ \\
\hline $30 \mathrm{mM}$ ammonium oxalate $(\mathrm{AO})$ & 11.08 & I & $15.81 \mathrm{~g}-\mathrm{j}$ & 20.77 b-e & 23.23 bc & $17.72 \mathrm{~B}$ \\
\hline 30 mM potassium oxalate (PO) & 11.06 & I & $15.19 \mathrm{~g}-\mathrm{k}$ & 19.98 b-f & 21.09 b-e & $16.83 \mathrm{~B}$ \\
\hline 50 ppm gibberellic acid $\left(\mathrm{GA}_{3}\right)$ & 11.08 & I & $16.57 \mathrm{f}-\mathrm{i}$ & 21.89 bcd & $24.08 b$ & $18.41 \mathrm{~B}$ \\
\hline $100 \mathrm{ppm} \mathrm{GA}_{3}$ & 10.74 & I & $15.31 \mathrm{~g}-\mathrm{j}$ & 20.06 b-f & 22.21 bcd & $17.08 \mathrm{~B}$ \\
\hline $30 \mathrm{mM}$ AO plus 50 ppm $\mathrm{GA}_{3}$ & 10.73 & I & $12.91 \mathrm{i}-\mathrm{I}$ & $16.04 \mathrm{~g}-\mathrm{k}$ & $17.52 \mathrm{~d}-\mathrm{h}$ & $14.30 \mathrm{C}$ \\
\hline $30 \mathrm{mM} \mathrm{AO}$ plus $100 \mathrm{ppm} \mathrm{GA_{3 }}$ & 10.44 & I & $12.89 \mathrm{i}-\mathrm{I}$ & 14.57 h-l & $16.14 \mathrm{f}-\mathrm{j}$ & $13.51 \mathrm{CD}$ \\
\hline $30 \mathrm{mM}$ PO plus 50 ppm GA & 10.73 & I & 12.73 i-I & $15.32 \mathrm{~g}-\mathrm{k}$ & 16.80 e-h & $13.90 \mathrm{CD}$ \\
\hline $30 \mathrm{mM}$ PO plus 100 ppm GA 3 & 10.43 & I & $11.90 \mathrm{jkl}$ & 13.57 h-I & $14.94 \mathrm{~g}-\mathrm{k}$ & $12.71 \mathrm{D}$ \\
\hline Means & 10.87 & $\mathrm{D}$ & $14.91 \mathrm{C}$ & $18.47 \mathrm{~B}$ & $20.87 \mathrm{~A}$ & \\
\hline
\end{tabular}

Means followed by the same letters season are not significantly different at level $\mathrm{P} \leq 0.05$ according to DMRT.

increase of water soluble galacturonic acids as a results of degradation of pectic substances by Hydrolytic enzymes (Reena, 2016), while a slight decline in this parameter at end of storage could be attributed to utilization of TSS in respiratory processes and degradation soluble sugars into alcohols and water (Kays, 1991).

Citric acid is the major organic acid in guava fruits and the faster reduction in acidity causing rise to a faster senescence (Nag et al., 2011). Higher TA, delayed an increment of TSS content and TSS/TA ratio in guavas treated with potassium or ammonium oxalate and gibberellic acid especially in combination may be due to slowing down the fruits ripening and delaying physiological ageing by reducing respiration rate and ethylene production as reported by (Zheng et al., 2007; Huang et al., 2013; Duguma et al., 2014; Reena, 2016).

Our results are in agreement with the findings of (Reena, 2016) on guavas, (Zheng et al., 2007 and Zheng et al., 2012.and Mohamed et al., 2016b) on mangoes. They reported that application of oxalic acid, oxalate and gibberellic acid treatments slowed the increase rate of TSS with an increase in TA content as compared to untreated treatment. In addition, they mentioned that TSS increased during storage period followed by a gradual decrease until end of storage, while TA decreased gradually with the advancement of storage period. Also, our results are in accordance with those reported by (Mohamed et al., 2016a, b) they claimed that treating 
mangoes fruits after harvest with oxalic acid or gibberellic acid significantly reduced TSS/TA ratio of citrus and mango fruits as compared to control (untreated) fruits. Furthermore, they added that TSS/TA ratio increased with the progress of storage time.

\section{CONCLUSION}

Generally, our experiment indicated that, the postharvest treatments of guava fruits strain 'Etmany' with $30 \mathrm{mM}$ potassium or ammonium oxalate and gibberellic acid at 50 or $100 \mathrm{ppm}$ either individual or in combinations significantly minimized physiological loss in weight, reduced decay incidence, maximized marketable percentage as well as fruit quality i.e. TSS, TA and vitamin C during cold storage. Finally, these treatments seemed to be promised since they are suppress postharvest deterioration of physico-chemical attributes as well as extend storage life of guava fruits strain 'Etmany' up to 21 days at $8^{\circ} \mathrm{C}$ plus 2 days shelf life at $18-23^{\circ} \mathrm{C}$.

\section{REFERENCES}

1. Anonymous. 2016. Ministry of Agriculture and Land Reclamation, Economic Affairs Sector, Bulletin of the Agricultural Statistics.

2. Boora, R.S., Dhaliwal, H.S. and Arora, N.K. 2016. Crop regulation in guava-A review. Agricultural Reviews, 37 (1), 1-9.

3. Duguma, T., Egigu, M.C. and Muthuswamy, M. 2014. The effects of gibberellic acid on quality and shelf life of banana (musa spp.). International Journal of Current Research and Reviews, 6(23), 63-69.

4. Hooley, R. 1994. Gibberellins: Perception, transduction and responses. Plant Molecular Biology, 26, 1529-1555.

5. Huang, H., Jing, G., Guo, L., Zhang, D., Yang, B., Duan, X., Ashraf, M. and Jiang, Y. 2013. Effect of oxalic acid on ripening attributes of banana fruit during storage. Postharvest Biology and Technology, 84, 22-27.

6. Kader, A.A. 2002. Postharvest technology of horticultural crops. Oakland: University of California, Division of agriculture and natural resources publication $3311,535 \mathrm{p}$.

7. Kays, S.J. 1991. Postharvest physiology of perishable plant products. AVI, Van Nostrand Reinhold, New York, USA 532 p.

8. Kumar, A. 2012. Importance for life 'Psidium guava' - review article. International Journal of Research in Pharmaceutical and Biomedical Science, 3(1), 137-143.

9. Mapson, C.W. 1970. Vitamins in fruits: stability of L-ascorbic acid. In: Biochemistry of Fruits and Their Products. Academic Press, London, pp. 376-387. 
10. McGuire, R.G. 1992. Reporting of objective colour measurements. HortScience, 27(12), 1254-1255.

11. Mercado-Silva, E., Benito-Bautista, P. and Garcia-Velasco, M.A. 1998. Fruit development, harvest index and ripening changes of guavas produced in central Mexico. Postharvest Biology and Technology, 13, 143-150.

12. Mohamed, M.A.A., Abd El-khalek, A.F. and Abdelaziz, A.M.R.A. 2016b. Increasing storability of mango cv. 'Zibda' fruits by 1-methylcyclopropene and gibberellic acid postharvest applications. Egyptian Journal of Horticulture, 43(2), 277-298.

13. Mohamed, M.A.A., Abd El-khalek, A.F., Elmehrat, H.G. and Gehan Mahmoud, A. 2016a. Nitric oxide, oxalic acid and hydrogen peroxide treatments to reduce decay and maintain postharvest quality of Valencia orange fruits during cold storage. Egyptian Journal of Horticulture, 43(1), 137-161.

14. M-STAT, 1993. A Microcomputer Program for the Design, Arrangement and Analysis of Agronomic Research Experiments. Michigan State University.

15. Nag, A.R., Chatterjee, D.D., Roy, T., Hossain, A.M.M.Z. and Haque, A. 2011. Study on chemical changes of different guava varieties during different ripening stage. Bangladesh Research Publication Journal, 6, 217-224.

16. Payasi, A., Mishra, N.N., Chaves, A.L.S. and Singh, R. 2009. Biochemistry of fruit softening: an overview. Physiology and Molecular Biology of Plants, 15, 103-113.

17. Reena, 2016. Role of gibberellic acid and calcium chloride in ripening related biochemical changes in guava (Psidium guajava L.) fruit. PhD thesis. Department of chemistry and biochemistry, College of Basic Science and Humanities CCS, Haryana Agricultural University, Hisar-125 004, 138 p.

18. Shewfelt, R.L. 1993. Measuring quality and maturity. Postharvest Handling: A Systems Approach, 99-124.

19. Vicente, A.R., Saladie, M., Rose, J.K.C. and Labavitch, J.M. 2007. The linkage between cell wall metabolism and fruit softening: looking to the future. Journal of the Science of Food and Agriculture, 87, 1435-1448.

20. Watkins, C. and Harman, J. 1981. Use of penetrometer to measure flesh firmness of fruit. Orchadist, N. Z., 14-16.

21. Zheng, X., Ye, L., Jiang, T., Jing, G. and Li, J. 2012. Limiting the deterioration of mango fruit during storage at room temperature by oxalate treatment. Food Chemistry, 130, 279-285.

22. Zheng, X.L., Tian, S.P., Gidley, M.J., Yue, H. and Li, B.Q. 2007. Effects of exogenous oxalic acid on ripening and decay incidence in mango fruit during storage at room temperature. Postharvest Biology and Technology, 45, 281-284. 


\title{
تاثير بعض معاملات ما بعد الحصاد على جودة ثمار الجوافة الشتوى سلالة العتمانى والقدرة التخزينية لها
}

تعتبر ثمار الجو افة من ثمار الفاكهه الكلايمكتيرية عالية القابلية للتف وذات عمرتسويقي

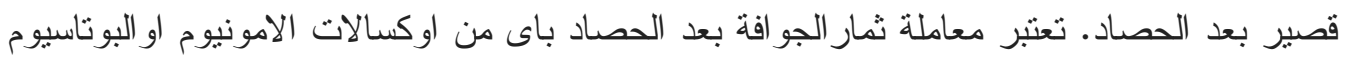
او حمض الجبريليك و التخزين عند درجة حرارة منخفضة من الطرق الفعالة لتعزيز القدرة التخزينية لثمار الجو افة دون حدوث تدهور لمواصفات الجودة. أجريت هذه التجربة خلال موسمي 10 . ب و و 17

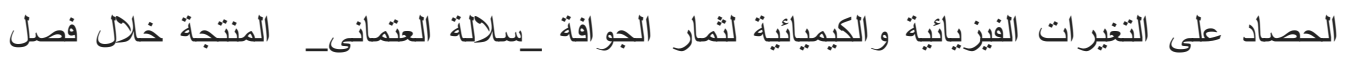

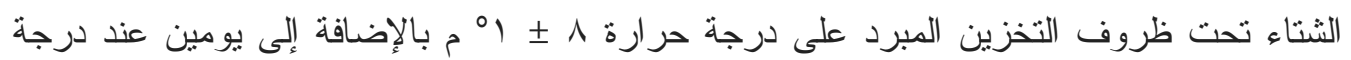

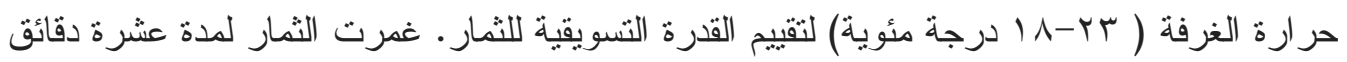

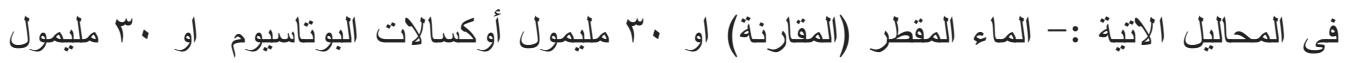

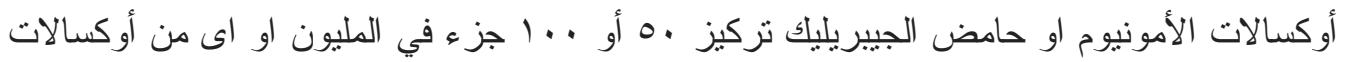

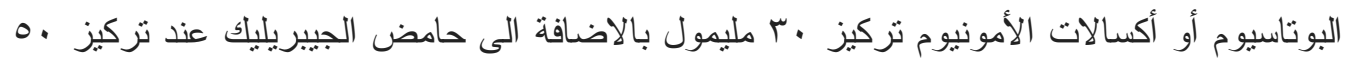

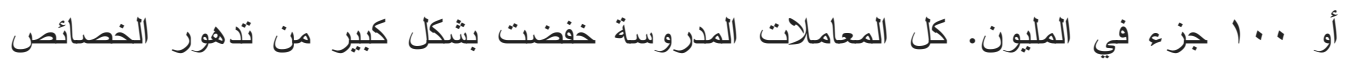
الفيزيائية و الكيميائية لثمار الجو افة الثنتوية "عتماني" مقارنة بالثمار غير المعاملة (المقارنة) خلال

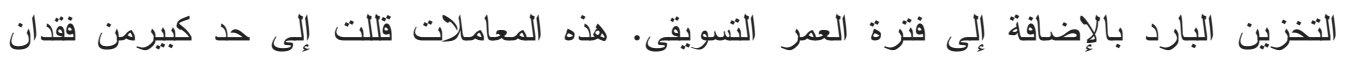
الوزن، وانخفاض حالات التذهور المرضى، وحسنت من نسبة الثمار القابلة للتسويق، والمحافظة

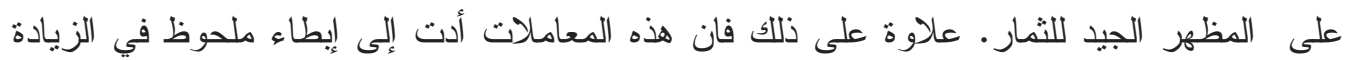
في محتوى المواد الصلبة الذائبة ونسبة المو اد الصلبة الذائبة الى الحموضة وكذللك خفضت من معدل تدهور محتوى الثمار من فيتامين C ونسبة الحموضة بالثمار مقارنة بثمار الكنترول خلال فترة التخزين البارد و العمر التسويقى. لتون.

كذلك كانت معاملات غمر الثمار قبل التخزين فى •ب مليمول من أوكسالات البوتاسيوم

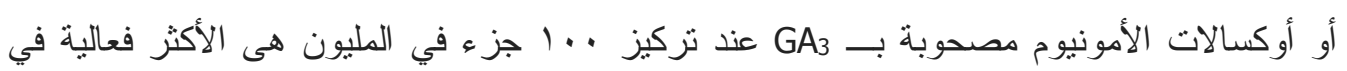

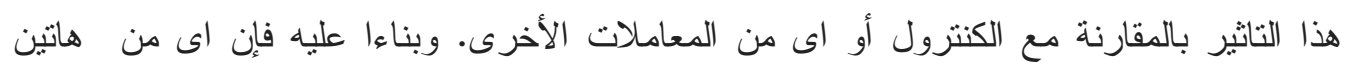

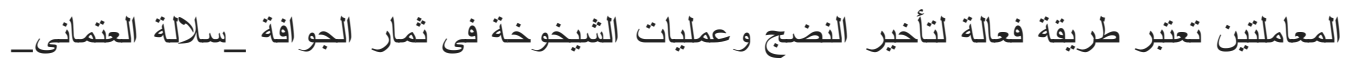

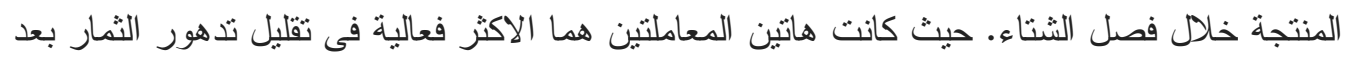

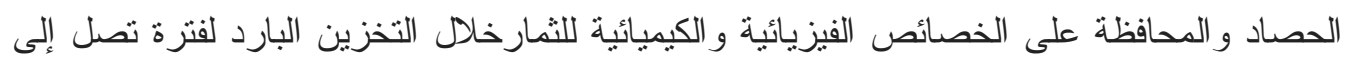

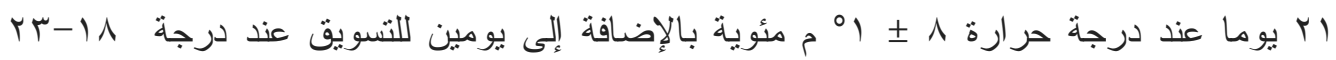

